\title{
INTRA MUROS. ZUR GESCHICHTE DES (ERZWUNGENEN) SCHWEIGENS DER LITERATUR
}

\author{
CZESŁAW KAROLAK
}

\begin{abstract}
In the article, an attempt has been made to analyze the systems of control of social communication based on the history of censorship in Germany. Apart from the historical context, reference has been made to the relationship of systems of censorship to modernization processes. In addition, the crucial role of legal awareness in society has been indicated. Within the scope of the present analysis, important methodological issues concerning research on forms, manifestations and effects of censorship related to the XXth century German literature have been considered.
\end{abstract}

Key words: censorship, publication control, control of social communication, modernization processes, legal awareness, social resistance, (seeming) freedom of expression

Literaturgeschichte kann einerseits betrachtet werden als Geschichte von Versuchen, jegliche literarische Produktion entweder zum Schweigen zu bringen oder sie in system- und ideologiekonformer Richtung zu beeinflussen, andererseits behandelt die einschlägige Fachliteratur das Zensurproblem (und dessen Folgen) als ein Register von Mitteln und Strategien, die von Schriftstellern und Publizisten unternommen wurden, um sich diesen Versuchen zu widersetzen oder ihren Konsequenzen vorzubeugen oder aber in extremen Fällen ihnen - auch physisch - zu entgehen. ${ }^{1}$ In diesen beiden Betrachtungsweisen, wie sie in zahlreichen primären und sekundären Quellen zum Thema Zensur präsentiert werden, scheint ein Aspekt (trotz dokumentarischer

\footnotetext{
${ }^{1}$ Der vorliegende Beitrag knüpft an die Einleitung zu meiner Dokumentation (Karolak, 2000, Hrsg.) an, vertieft zugleich einige dort behandelte Aspekte und erweitert sie um Fragestellungen zur Methodologie der Zensurforschung.
} 
Verlässlichkeit, durch die sie sich auszeichnen) relativ wenig berücksichtigt zu werden: es ist die Bedeutung der sozialen Normen und ihres Wandels, in dessen (und deren) Kontext die Zensur als Institution und gesellschaftliche Praxis wirkte.

So kann man also die Geschichte der Zensur einerseits aus der Perspektive der Tatsachen (sprich: der Eingriffe) betrachten, und diese Betrachtungsweise ist relativ oft angewandt worden. Ein kumulatives manchmal gar ,anekdotisches“ Exponieren der Ingerenz in den Prozess literarischer Kommunikation ist für diese Betrachtungsweise charakteristisch ${ }^{2}$. Andererseits ist die Geschichte der Zensur ein Gebiet, das man aus der Perspektive der Rechtsgeschichte und der Geschichte des Rechtsbewußtseins betrachten kann; dieser Ansatz ist für die Zensurforschung der letzten Jahrzehnte charakteristisch ${ }^{3}$. Diese Perspektive bedeutet zugleich die Notwendigkeit, das historische Beziehungsgeflecht zwischen Zensursystemen und den jeweiligen Rechtssystemen zu berücksichtigen, weil diese den Begriff, das Rechtsbewusstsein in puncto Zensur und deren Folgen beeinflusst haben; damit stecken diese Faktoren den methodologischen Horizont synchronischer und diachronischer Zensurforschung ab.

\section{Zensurgeschichte und Modernisierungsprozesse}

Bereits im 16. Jahrhundert entwickelte sich in Deutschland ein Rechtssystem, das Produktions- und Distributionsbedingungen von Druckerzeugnissen bestimmte ${ }^{4}$. Damit sind im Zuge der Modernisierung des Rechts Regelungen eingeführt worden, die für das damalige System kirchlicher und staatlicher Machtausübung wichtige Folgen hatten. Eine dieser Folgen war das Wormser Edikt (1521), das kritische Äußerungen über den Papst und die damalige kirchliche Hierarchie verboten hat. Ein wesentliches Novum dieser rechtlichen Regelung war (,konsequenterweise“) die Einführung der Präventivzensur.

Die Geschichte der Zensur in Deutschland ist ein spezifisches Register von rechtlichen Gegensätzen, die die Funktion der Zensur als Institution beeinflusst haben. Wenn wir unter diesem Aspekt die Rechtslage im 16. und 17. Jahrhundert betrachten, dann sind in erster Linie Unterschiede zwischen dem zentralistischen System der Kirchenzensur bei den Katholiken gegenüber der regionalen Struktur der protestantischen Kirche als Modernisierungspotential zu qualifizieren, dessen Wirkung in zwei gegensätzlichen Tendenzen bestand:

\footnotetext{
${ }^{2}$ Umfangreiche Dokumentationsarbeiten, die in diesem Zusammenhang genannt werden können, stammen von Houben, 1918; 1924; 1928.

${ }^{3}$ Hier kann auf weitere Quellen verwiesen werden: Petersen, 1988; 1995; Brockmeier, 1996; Bark, 1997.

${ }^{4}$ Eisenhardt, S. 1f. In: Göpfert/Weyrauch, 1988.
} 
Während die Territorialstaaten im Reich ihre Zensurinstitutionen ausbauten, führte der Liberalismus der Universitäten und die den Druckern gewährte Freiheit in den benachbarten Niederlanden dazu, daß das Land ,zu einem Zentrum der Gelehrsamkeit und des Journalismus im Europa des 17. Jahrhunderts' ${ }^{6}$ wurde $^{5}$

Im Laufe des 18. Jahrhunderts hat sich - was den Gegenstand der Zensur betrifft - ein wesentlicher Wandel vollzogen - der Übergang von der Zensierung religiöser Schriften zur Kontrolle und Indizierung von Publikationen auf ihre politischen Inhalte hin. Dieser Übergang wurde von der Französischen Revolution wesentlich intensiviert und beschleunigt ${ }^{6}$.

Diese Situation bleibt praktisch unverändert bis Ende des 18. Jahrhunderts ${ }^{7}-$ trotz spektakulärer Bemühungen und Stellungnahmen damaliger Autoritäten, die das Ziel verfolgten, diese Situation zu ändern. Um 1788 schrieb Christoph Martin Wieland:

„...wissen wir nicht aus Erfahrung, daß in Ländern, wo eine willkürliche Censur herrscht, gerade die vortrefflichsten Bücher die ersten sind, die in den Index librorum prohibitorum gesetzt werden?... Ich gestehe, daß ich von der Notwendigkeit einer eignen Büchercensur in einem wohlpolizierten Staate, nicht sehr überzeugt bin. Warum bestellt man nicht auch besondere Aufpasser, die dahin sehen, daß Niemand sich betrinken, oder dem andern eine Ohrfeige geben, oder seine Taschenuhr mausen, oder irgend ein anderes Gebot im Dekálogus übertreten könne. Man läßt es ganz ruhig darauf ankommen, und begnügt sich, den wirklichen Übertreter zu bestrafen, wenn er nach rechtlicher Untersuchung des Verbrechens überwiesen worden ist. Warum hält man es mit den schriftstellerischen Verbrechen nicht eben so? Der Censor, dem ein criminelles Manuscript in die Hände kommt, kann und darf doch (wenige Fälle ausgenommen) nichts weiter tun als den Druck verbieten? Und dieses Verbots ungeachtet, wird es irgendwo gedruckt und als Contrebande in den Staat hineingeschwärzt werden. Wozu also die Censur?“8

Zum „Modernisierungsaspekt” der Zensur-(Geschichte) in Deutschland - besonders was Publikationsfreiheit betrifft - muss angemerkt werden, daß man sich erst in der zweiten Hälfte des 18. Jahrhunderts für den rechtlichen Aspekt der Zensur zu interessieren begann. Der aus Breslau stammende Jurist Carl Gottlieb Svarez (1746-1798) hat als erster in Deutschland nachgewiesen, daß die Tätigkeit der Zensur einen Eingriff in das Freiheitsrecht bedeutet. In einigen anderen Ländern jedoch (unter anderem in Frankreich und besonders in den USA) ist man schon früher auf die rechtlichen Aspekte des Zensurproblems aufmerksam geworden. Generell - und nicht nur in bezug auf Deutschland - muß darauf hingewiesen werden, daß sich das Rechtsbewußtsein bezüglich der Zensur relativ spät herauskristallisiert hat; im

\footnotetext{
${ }^{5}$ Schütz, S. 23 f.

${ }^{6}$ Eisenhardt, op.cit., S. 19).

${ }^{7}$ ebda., S. 3.

${ }^{8}$ Wieland, 1967, S. 573 f.
} 
Vergleich mit dem Rechtsbewußtsein bezüglich des Eigentumsrechts, das bereits in der 2. Hälfte des 17. Jahrhunderts (durch John Locke) seinen Platz im Rechtssystem erhalten hat, hat sich das Rechtsbewußtsein bezüglich der Zensur (also der Äußerungsfreiheit) wesentlich später entwickelt.

Als Symptom und Folge dieses Prozesses kann eine allmähliche Liberalisierung der Theaterzensur (um die Mitte des 18. Jahrhunderts) angesehen werden. Das bezieht sich zum Teil auf die damalige Presse. Dieser Liberalisierungsproze $\beta$ fand in der ersten Hälfte des 19. Jahrhunderts vor allem in Österreich statt, aber zugleich bemühte sich die dortige politische Führung und die Verwaltung der Fürstenstaaten im Deutschen Bund darum, Rechtswege zu finden und Mechanismen zu schaffen, die den Liberalisierungsprozess verhindern könnten. Die Karlsbader Beschlüsse vom August 1819 waren ein Ausdruck dieser Bestrebungen. Ihre antiemanzipatorische Wirkung erfolgte einerseits auf dem Weg einer rechtlichen Formalisierung (es wurden beispielsweise der Umfang und die Art der zu zensierenden Publikationen genau festgelegt), andererseits macht sich eine (wie Dieter Breuer hervorhebt), spezifisch deutsche" Tendenz" bemerkbar: Hindernisse, die aus territorialer Zersplitterung resultierten, Kommunikationsschwierigkeiten, die für die damals aktiven Schriftsteller und Publizisten ein Problem darstellten, als „Stimulatoren“ des literarischen Lebens zu nützen. Diese Tendenz (Breuer spricht in diesem Zusammenhang von einer deutschen Besonderheit) reicht in unsere Gegenwart hinein:

„Das Nebeneinander von Territorialstaaten unterschiedlicher ideologischer Ausrichtung im Reichsgebiet hat insgesamt zwar die Schriftsteller sehr behindert, aber mindestens ebenso die Zensur, ja, es hat letztlich sogar stimulierende Wirkungen auf das geistigliterarische Leben gehabt, zumal Zensurfreiheit...nicht zur Wahl stand und steht, sondern nur ein Mehr oder Weniger an Zensur. Verglichen mit den großen zentralistischen Einheitsstaaten Europas, kann man in diesem Nebeneinander sogar einen Vorzug der vielgeschmähten „deutschen Kleinstaaterei“ erblicken, und dies bis auf den heutigen Tag jedenfalls unter dem Aspekt literarischer Zensur; man denke nur an die Publikationsmöglichkeiten der vielen Exulanten, ja Ausgebürgerten im jeweils anderen deutschen Staat. Heine konnte in Preußen und in den von Preußen kontrollierten Staaten nicht publizieren, aber in Hamburg, Anastasius Grün ${ }^{9}$ nicht in Österreich, aber in Sachsen, Reiner Kunze und Wolf Biermann nicht in der DDR, aber in der Bundesrepublik. Niemals hat sich die Zensur so verheerend auf das geistig-literarische Leben auswirken können wie im zentralistischen deutschen Einheitsstaat, als dieser in der ersten Hälfte des 20. Jahrhunderts für zwölf Jahre Wirklichkeit wurde. Diese Besonderheiten der literarischen Zensur wie des literarischen Lebens in Deutschland machen eine historische Darstellung vielleicht komplizierter als gemeinhin angenommen, aber sie entschädigen den Betrachter durch größere Wirklichkeitsnähe.“ ${ }^{10}$

\footnotetext{
${ }^{9}$ Österreichischer Dichter (1806-1876); in seinen Gedichten verbreitete er Ideen des bürgerlichen Liberalismus.

${ }^{10}$ Breuer, 1982, S. $21 \mathrm{f}$.
} 
Ein gemeinsames Merkmal der Zensur (-Systeme) zumindest im 19. Jahrhundert (man kann in diesem Zusammen sogar von einem psychologischen Mechanismus sprechen) war die Präferenz moralischer Argumente in einer Ersatzfunktion, um einzelne Eingriffe zu rechtfertigen. Dies hat sich besonders im Bereich der Theaterzensur ausgewirkt. Hier wirkte ein Konstruktionsmechanismus von Ersatzargumenten für Zensureingriffe, auf den Jost Hermand und Hans J. Schütz verweisen. Als Beispiel führt Schütz die Kampagne gegen jungdeutsche Autoren an, besonders gegen Karl Gutzkow.

Das Metternich-Regime und seine Anhänger deklarierten nur ihr Interesse daran, die Zuschauer und Leser vor „unmoralischen Inhalten“ zu schützen; im Grunde genommen war es ein Kampf darum, was auch zu den Hauptfunktionen der Zensur gehört: die Kommunikationskanäle in der Gesellschaft zu blockieren, die es den breiteren gesellschaftlichen Kreisen ermöglichen könnten, Probleme der Politik zur Angelegenheit der ganzen Gesellschaft zu machen und sie als solche zu betrachten. ${ }^{11}$ Diese Strategie hatte auch deshalb Erfolgschancen, weil die Staatsschützer des Metternich-Regimes Hilfe und Unterstützung von Intellektuellen aus konservativen Kreisen erhalten haben, allen voran von dem Journalisten und Literaturhistoriker Wolfgang Menzel, der im Herbst 1835 im Stuttgarter „Literaturblatt" wütende Attacken gegen die jungdeutschen Autoren veröffentlichte, deren literarische und publizistische Produktion er als Inbegriff ,undeutscher“ Art und „Lasterschule der Unzucht" bezeichnete.

Vor diesem historischen Hintergrund erscheint das Wilhelminische Deutschland - was die Geschichte der Zensur betrifft - als Anfang eines modernen Staates und damit auch als historische Ausgangsbasis für eine gegenwartsbezogene Fokussierung der Zensurforschung. Eines der Modernisierungsattribute ist in diesem Zusammenhang das Bestreben, das System und die Institutionen der Zensur auf rechtliche Grundlagen zu stützen. ${ }^{12}$ Dieser Prozess scheint von gesellschaftlichen Formationen, politischen Präferenzen und Ideologien relativ unabhängig zu sein. Dies bedeutet aber zugleich, daß damit eine Selektivität der Vorgehensweisen und (dadurch auch) der Auswirkungen und Folgen der Zensur verbunden sein kann. So weist Klaus Petersen auf den selektiven Charakter der Tätigkeit der Zensurinstanzen in der Weimarer Republik hin und meint damit eine evidente „Asymmetrie“ zwischen den damaligen juristischen Prozeduren bezüglich der linken Literatur und Publizistik gegenüber dem faschistischen bzw. nationalsozialistischen Schrifttum, das verhältnismäßig „mild“ behandelt wurde. Bekanntlich haben die Gerichte der Weimarer Republik - wenn überhaupt - meistens nur einzelne „Delikte“, das heißt in diesem Fall, Publikationen, die z.B. zur Gewalt aufgerufen haben, eben als einzelne „Fälle“ mit Sanktionen belegt. Dagegen ging es bei Verfahren gegen linke

\footnotetext{
${ }^{11}$ Schütz, op.cit., S. 124.

${ }^{12}$ Vgl. Eisenhardt, op.cit., s. 34.
} 
Schriftsteller und Publizisten um die Verfolgung und Bekämpfung einer Gesinnung. ${ }^{13}$

Sind aber die inneren Widersprüche, die Klaus Petersen in seinem Buch „Zensur in der Weimarer Republik" (1995) hervorhebt, nur ein Symptom bestehender politisch / staatspolitisch nachvollziehbarer Inkonsequenzen in der Performanz des Rechts oder sind sie mehr als nur ein derartiges Symptom? Bei der Beantwortung dieser Frage könnte ein bereits in den 60er Jahren formulierter Vorschlag der Soziologin Ulla Otto (in späteren Veröffentlichungen Otto-Fölsing) behilflich sein. Otto knüpft an die Theorie über den Kreislauf der Eliten von Vilfredo Pareto (1848-1923) an, dem italienischen Wirtschaftswissenschaftler und Soziologen), wobei Pareto den Begriff „Elite“ wertneutral versteht (es sind die „Besten“, „Aktivsten“ einer Handlungskategorie - nicht nur Politiker oder politisch Herrschende), die selbst in revolutionären Umwälzungen von Reserve-Eliten und nicht von einer „Masse“ ersetzt werden, wenn auch ideologisch etwas anderes artikuliert wird, um schein-logische Rationalisierungen bestehender Verhältnisse zu konstruieren. Ulla Otto weist auf die wichtige und deutliche Relation hin zwischen einem systemimmanenten Schutz axiologischer Kategorien, die in einer Gesellschaft funktionieren und dem Bestreben von Machteliten, im Rahmen des gesellschaftlichen System die Machtposition und interessen zu bewahren. Die literarische Zensur basiert, so Otto, ,auf der Erkenntnis von der Notwendigkeit der Einflußnahme auf die Meinungen und das Denken der Beherrschten zur Machtbehauptung der Herrschenden. Ihr spezifischer Ansatzpunkt ist dabei die Disziplin der Literatur als potentielles Gefahrenmoment für die Herrschaftsausübenden... In diesem Sinne ist die literarische Zensur ein Instrument der sozialen Kontrolle, das hauptsächlich gegen solche Faktoren gerichtet ist, die einen Wandel des betreffenden sozialen Systems herbeiführen könnten“. ${ }^{14}$

Das Beispiel der ,asymmetrischen Zensur“, wie es von Klaus Petersen charakterisiert wurde (mit Sicherheit kein einziger derartiger „Fall“ in der Zensurgeschichte) deutet auf die Notwendigkeit hin, Differenzen im Bereich der Funktionen der Zensur sowie Richtungen und Perspektiven der Zensurforschung neu zu definieren.

\section{Der „funktionale Ansatz“ der Zensurforschung}

Bis in die 2. Hälfte des 20. Jahrhunderts hinein hat in der Zensurforschung ein kumulativ-anekdotischer Narrationstypus dominiert: eine mehr oder weniger lineare Darstellung und Analyse einzelner „Zensurfälle“ konnte zwar als DiagnoseInstrument angewandt werden - zur Rekonstruktion der Motive einzelner Eingriffe und des politischen Kontextes, in dem bestimmte Maßnahmen gegen literarische

\footnotetext{
${ }^{13}$ Petersen, 1995, S. 289 f.

${ }^{14}$ Otto, 1968, S. 138 f.
} 
oder publizistische Äußerungen ergriffen wurden - aber eine ideologie-übergreifende Anwendung solcher Diagnose-Instrumente war kaum möglich. Ulla Otto sieht eine wichtige Aufgabe der Zensurforschung in einer systemübergreifenden, nicht auf einzelne ideologische Optionen ausgerichteten Analyse der Zensursysteme. Diese Aufgabe kann praktisch nur dann als realisierbar angesehen werden, wenn man sie im Kontext eines funktionalen Literaturbegriffs betrachtet. Otto unterscheidet wichtige soziale Aspekte der „Rolle der Literatur in der Gesellschaft“, die mit der Präsenz bestimmter Elemente in literarischen Werken verbunden sind. Sie hebt das ,rezeptive“, das „reflexive“, das ,ideologische“, das „,kommunikative“, „,normative“, ,aktivierende“ und „revolutionäre Element der Literatur“ hervor. ${ }^{15}$ Diese Elemente beziehen sich auf die gesamte „Bandbreite“ der Literaturfunktionen, insbesondere auf meinungsbildende Potenzen, deren Wirksamkeit verschiedene Ebenen des sozialen Lebens betreffen kann. Daher muß die Beschäftigung mit historischen Phänomenen der Literaturzensur gerade die funktionalen Aspekte berücksichtigen. Die Feststellung (Otto:8), daß Literatur meistens über eine „rein soziale Deskription“ hinausgeht ist bei Otto ein wesentlicher „Orientierungspunkt“ für die Zensurforschung; hier wird aber auch das Bestreben nach deutlich, den methodologischen Ansatz der Zensurforschung an „systemübergreifenden“, paradigmatischen Fragestellungen zu orientieren. „Da die Literatur als geschriebenes Wort unter bestimmten Vorzeichen auf nachhaltige Weise Meinungen auszurichten, zu prägen und zu verbreiten in der Lage ist - und das unter Umständen gegen das bestehende System, gleich welcher Art es ist - mußte sie historisch und faktisch von dem Augenblick ihres Erscheinens an den Versuchen der herrschenden Klasse unterliegen, sie zur systemkonformen Waffe auszubilden und, wenn nötig, im gewünschten Sinne zu beschneiden.“ ${ }^{16}$

Von diesem Standpunkt aus gesehen, kann man feststellen, daß sogenannte „Zensurargumente“ (religiöser Art - z.B. „Gotteslästerung“; politische Argumente „Staatsgefährdung“, „Subversion“ bzw. moralische Argumente - „Jugendgefährdung“, „Pornographie“) eigentlich nur formal der Rechtfertigung von Zensureingriffen gedient haben; sie stellten in Laufe der Geschichte vor allem Versuche dar, axiologische Systeme und den sozialgruppenspezifischen (oft) emotionalen Kontext, in dem ihre Exponenten zur Sprache gebracht werden, zu besetzen und zu beeinflussen. Nach Pareto sind es typische Derivationen, die von einer herrschenden Minderheit/Elite zum Schutz ihrer eigenen Interessen und zur Vortäuschung einer Gemeinsamkeit dieser Interessen (bis hin zu der Andersonschen Kategorie der imagined communities) artikuliert werden. Dass die herrschende Minderheit sich dadurch wenigstens bestimmter Teile der Wertsysteme bemächtigt und dies mit dem Argument rechtfertigt, die Interessen der Mehrheit dadurch zu schützen, ist nach Pareto (und nach Ulla Otto) ein Beispiel für zweckgebundene Derivationen. Hans J. Schütz

\footnotetext{
15 ebda., S. 8-10.

${ }^{16}$ ebda., S. 137.
} 
weist auf unpräzise und allgemeine Formulierungen der Zensurargumente hin wie „Einheit und Opferbereitschaft für große nationale Ziele“; diese Globalität und zugleich auch Unschärfe der Argumente hat es den Zensoren ermöglicht, Entscheidungen gegen Publikationen zu treffen, die oder deren Autoren aufgrund ihrer kritischen (oft gesellschaftskritischen oder pazifistischen) Tendenz unbeliebt waren. ${ }^{17}$

Ein vergleichbarer methodologischer Ansatz der Zensurforschung (vergleichbar mit den Positionen der Theorie über den Kreislauf der Eliten von Pareto, die Ulla Otto ihrer „Zensurtheorie“ zugrunde gelegt hat), stammt von Dieter Breuer. Seine Vorschläge sind systemtheoretisch orientiert; das bedeutet in diesem Kontext (und es ist eine wichtige These von Breuer), daß Zensursysteme als Regulativ der Grundfunktionen des Staates betrachtet werden können. Breuer stellt gerade die regulativen Funktionen der Zensur in den Vordergrund; er knüpft an die Arbeit von Ulla Otto an, unterstreicht aber vor allem den Aspekt der Stabilisierung von Normen und Wertsystemen durch Zensur, weil Normen und Wertsysteme ein als Objekt ,poetischer Antithesen“ der Literatur funktionieren können. ${ }^{18}$

Auf eine symptomatische (um nicht zu sagen: paradigmatische) Art und Weise bezieht sich die „normenzentrierte“ Konzeption von Dieter Breuer auf mögliche Vorschläge einer komplexeren interdisziplinären Zensurforschung. So schlägt er „Themenbereiche“ für die Zensurforschung vor (bzw. geht auf Vorschläge anderer Zensurforscher - Ulla Otto, Michael Kienzle und Dirk Mende - ein), die die Relationen zwischen Zensur und Eigentumsrecht betreffen (Zensur und Kapital), Zensur und Strafrecht, Zensur und Medien, Bibliotheken, Gewerkschaften Militär sowie die Relation zwischen Zensursystemen und Kirchen, die historisch gesehen, einen besonderen Schwerpunkt der Zensurforschung bildet (einige wichtige Problembereiche dieser Art behandeln Michael Kienzle und Dirk Mende in ihrer Dokumentation Zensur in der BRD. Fakten und Analysen ${ }^{19}$ ).

Breuer vertritt die Auffassung, daß der Katalog von Fragen und Problembereichen, mit denen die Zensurforschung konfrontiert wird, zugleich ein Katalog von sozialen Normen ist, weil jeder dieser Bereiche, die potentieller Gegenstand öffentlich artikulierter Ansichten sein können, durch Prozesse und Bestrebungen der Normbildung gekennzeichnet ist. Deshalb ist hier auch ein Konfliktpotential vorhanden, das mit Prozessen des Normenwandels verbunden ist.

„Kein Bereich der öffentlichen Meinungsäußerung ist frei von Normen, keiner ist aber auch frei von Konflikten, von Kämpfen im notwendigen Normenwandel, von Konflikten

\footnotetext{
${ }^{17}$ Hans J. Schütz spricht in diesem Zusammenhang vom Mangel an Präzision und weist auf Globalität und Pauschalität in der Formulierung politischer Ziele hin, (z.B.: „Einheit und Opferbereitschaft für große nationale Ziele“) und bemerkt, dass die Zensoren solche globalen und unpräzisen Statements benutzt haben, um gegen Bücher und unbeliebte Autoren vorzugehen. Siehe Schütz, S. 135-155.

${ }^{18}$ Breuer, op.cit., S. 17.

${ }^{19}$ Kienzle/Mende, 1980.
} 
durch sich verfestigende Normen. Das alles kann aber unmöglich unser Aufgabenbereich sein. Der Zensurbegriff des Literarhistorikers wird sich, in Kenntnis der Breite der Konflikte um Normen, auf die autoritäre Kontrolle der Künstler und hier vor allem der Schriftsteller zu beschränken haben. Das heißt aber, wir werden die Konflikte um die jeweils geltenden Normen von ' Kunst und Dichtung, von künstlerischer Freiheit zu sammeln und zu erklären haben, näherhin die Kontrolle und die Kontrollversuche des dichterischen Worts: die literarische Zensur. ${ }^{\text {‘20 }}$

Trotz dieser Einschränkungen kann die Forschungsrichtung, die von Dieter Breuer vorgeschlagen wird, als politologisch angesehen werden; die im Umfeld der Zensurforschung befindlichen Objekte wie Prozesse der Meinungsbildung und Manipulation und ihre sozialen und politischen Konsequenzen weisen in diese Richtung. ${ }^{21}$ Das Problem der Zensurfolgen betrachtet Breuer ebenfalls von einem ,funktionalen" Standpunkt aus und anknüpfend an Ulla Otto vertritt er die Auffassung, daß die Zensur keinesfalls für die Eliminierung von Büchern und anderen Publikationen verantwortlich sei; wenn sie aus dem Kommunikationsumlauf „,verschwinden“, dann liegt der Grund dafür im Verlust ihrer sozialen Wirkung ${ }^{22}$ und nicht in der Effizienz der Zensurmaßnahmen. Dies stellt jedoch ein komplexeres Problem dar, dem wir uns noch später zuwenden werden.

Mit einem vergleichbaren Forschungsansatz (insofern vergleichbar, als hier auch Fragen der Rezeption und Rezeptionsbedingungen eine Rolle spielen) haben wir es zu tun, wenn als Ausgangspunkt die Relation zwischen Zensur und Kanonbildung angenommen wird. Jeder Kanon bedeutet praktisch eine Einengung der literarischen Tradition. Relationen zu Zensursystemen sind vor allem aufgrund der Tatsache möglich, daß jeder Kanon durch Trennstriche und Eliminierung zustande kommt, woraus sich dann auch bestimmte negative Konsequenzen für die außerhalb des Kanons befindliche Literatur ergeben: Tabuisierung und Invalidierung, die sich als Schwächung und Einschränkung der Wirkung dieser Literatur und/oder Marginalisierung ihrer Tradition manifestiert. ${ }^{23}$

Als Beispiel für praktische Folgen dieser Art, die auch von einer institutionellen Basis ausgehen können, können Literaturgeschichtsbücher aus dem Dritten Reich angesehen werden; es wurden, besonders in den 30er Jahren relativ viele Literaturgeschichtsbücher verfaßt, die - gerade wegen selektiver und marginalisierender Tendenzen im Umgang mit literarischer Tradition der Tätigkeit der Zensur funktional zugeordnet werden könnten. Orlowski bezeichnet diese Literaturgeschichtsschreibung $^{24}$ als (präsentistische) „Legitimierung der gesamten 'Deutschen Bewe-

\footnotetext{
${ }^{20}$ Breuer, op. cit., S. 13.

${ }^{21}$ Siehe Tullock, 1968, S. 1265-1267.

${ }^{22}$ Breuer, op.cit., S. 21. Siehe auch: Otto, op. cit. S. 128.

${ }^{23}$ Assmann/Assmann, 1987, S. 11.

${ }^{24}$ Linden, 1940; Fechter, 1941; Lübbe/Lohrmann, 1943; Koch, 1935; Bartels, 1942.
} 
gung' insbesondere der 'Deutschen Revolution' 1933“. ${ }^{25}$ Es war selbstverständlich ein Teil komplexer kulturpolitischer Maßnahmen, denen bestimmte Entscheidungen institutioneller Art gefolgt sind, bzw. institutionelle Entscheidungen konnten sich daran orientieren (insbesondere Entscheidungen darüber, was gedruckt und was nicht gedruckt, was wiederaufgelegt und was nicht wiederaufgelegt werden soll.

Gerade der institutionelle Zugriff (oder zumindest die rechtlich abgesicherte Wahrscheinlichkeit eines institutionellen Zugriffs), ist ein Faktor, der die Performanz der Zensur und ihre Formen als Objekte konkreter Diagnosen durch die Zensurforschung erscheinen läßt. Läßt sich diese Referenz (der Hinweis auf die Möglichkeit des institutionelle Zugriffs) nicht (mehr) rekonstruieren, dann haben wir es mit „dehnbaren“, unscharfen Begriffen zu tun wie dem der „regimekritischen Literatur im Dritten Reich“'26.

Das Problem der Relation zwischen Kanon und Zensur betrifft nicht nur den (semantisch definierbaren) Bereich dieser Begriffe, in dem vor allem der Aspekt der Normbildung im Vordergrund steht. Wichtig ist hier auch die Kategorie die als „kanonisches Subjekt" bezeichnet werden kann und eine normbildende Grundlage des Kanons bildet. Aleida und Jan Assmann schlagen eine begriffliche Differenzierung vor (und knüpfen dabei an die Herrschaftstypen von Max Weber - besonders an den „charismatischen“ Typus - an) zwischen einem „Kanon von oben“ und einem „Kanon von unten“ ${ }^{27}$ Sie betrachten die Kanonbildung als traditionsstabilisierende Faktoren. Sie berufen sich dabei auf den Stellenwert der Tradition in der Kultur von China und Ägypten und weisen auf das historisch fundierte Bestreben der Herrschenden hin, den „Kanon von oben“ auf dem Fundament eines „Kanons von unten“ aufzubauen (oder zumindest den Eindruck davon zu erwecken). Der Kanon von unten erscheint dann als Quelle charismatischer Sinngebung. Gerade dieses Bestreben bestimmt ihrer Ansicht nach ,die strukturelle Verkoppelung von Kanon und Zensur". Diese Beziehungen werden von Aleida und Jan Assmann folgendermaßen charakterisiert:

„Selbstverständlich sind nicht alle Zensurentscheidungen auf bestimmte Kanon-Werte gestützt. Es gibt eine andere Zensur, die rein kasuistisch in irrationalen Willkürakten als schiere "Notwehr der Macht" zur Erscheinung kommt. Und es gibt eine Zensur, die, wesentlich systematischer aber ebenfalls ohne Rückkoppelung an einen Kanon, der Macht ihre Ungreifbarkeit und Stabilität sichert, indem sie die Vergangenheit auslöscht und das kulturelle Gedächtnis auf die herrschende Gegenwart reduziert. Dieses wohl ehrgeizigste Zensur-Unternehmen ist in despotischen (z.B. im China des Qin Shi Huangdi, oder im Rom des Tiberius)...und totalitären (exemplarisch dargestellt in Orwells 1984) Herrschaftsformen praktiziert worden...All das heißt jedoch nicht, daß die kanongestützte Zensur darum auch schon legitimer und menschlicher wäre. Aber durch Eingrenzung des

\footnotetext{
${ }^{25}$ Orłowski, 1983, S. 59; auch: Ketelsen, 1992, S. 72-93.

${ }^{26}$ Denk, 1995.

${ }^{27}$ Assmann, op. cit., S. 22 f.
} 
Verbindlichen und Ausgrenzung alles anderen entsteht doch ein erkennbarer und verhältnismäßig eindeutiger Dispositionsraum. Der Kanon motiviert die Zensur, aber er kontrolliert sie auch, und jeder zensorische Akt im Schatten eines Kanons ist auch ein Akt der Selbstbeschränkung. “ 28

Interessante Anregungen können sich für die Zensurforschung aus kommunikationstheoretischen und diskursanalytischen Ansätzen ergeben; die Tätigkeit und vor allem die Folgen der Zensur können als Symptome „,kommunikativer Entropie“ und allgemeiner Reglementierung der Teilnahme des Individuums an den Prozessen sozialer Kommunikation angesehen werden. Wie Klaus Kanzog bemerkt ${ }^{29}$, funktionieren Entropie und Reglementierung als Attribute der Zensurtätigkeit, und dies bedeutet, dass für den Zensurforscher notwendigerweise auch der Kontext dieser Prozesse, vor allem die Maßnahmen zur ihrer Einschränkung oder gar Verhinderung von besonderer Relevanz sind ${ }^{30}$.

In diesem Zusammenhang kann auf einen Aufsatz von Franz Hebel verwiesen werden; der Verfasser repräsentiert den Bereich der (fremdphilologischen, interkulturell orientierten) Literaturdidaktik ${ }^{31}$. Hebel spricht von Tendenzen zur Inkorporation, die eine ,zentrale Kultur“ kennzeichnet und davon dass diese auf kulturelle Tätigkeiten trifft, die nach zwei Aspekten zu unterscheiden sind:

- als alternativ zur herrschenden (dominierenden) Kultur oder

- als oppositionell zur herrschenden Kultur

Nach ihrem temporären Aspekt können sie als „residuale“ (traditionsbedingte) oder neue (neu entstandene) Elemente der jeweils gegenwärtigen herrschenden Kultur angesehen werden.

Bei verstärkter Tendenz zur Inkorporation, so Hebel, betrachtet die herrschende Kultur den Unterschied zwischen alternativen und oppositionellen kulturellen als unwesentlich.

Auch in solchen Fällen spielt die Zensur, die von Hebel in diesem Zusammenhang explizit genannt wird, eine wesentliche Rolle, ${ }^{32}$ gerade, wenn es um das Prob-

${ }^{28}$ Assmann, op. cit. S. 21. In der Fachliteratur wird auch auf eine phänomenologische Richtung der Zensurforschung verwiesen; siehe Hobohm, Hans-Christoph. 1992. Hier wird vor allem die generelle Frage gestellt, ob Literatur überhaupt imstande ist, eine Bedrohung der sozialen oder moralischen Normen darzustellen. Siehe auch Armin Biermann, 1987. In: Assmann, op. cit., S. 212-226. Mit diesem Problem setzt sich unter anderem auch Wilhelm Emrich. 1968 auseinander, S. 215-220.

${ }^{29}$ Kanzog, 1984, S. 998-1049.

${ }^{30}$ Auf diese Notwendigkeit haben bereits Friedrich A. Kittler und Horst Turk (1977) im Vorwort ihrer Untersuchung Urszenen. Literaturwissenschaft als Diskursanalyse und Diskurskritik. Suhrkamp Verlag, Frankfurt a.M., S. 9-43. verwiesen. Es treten hier gewisse Parallelen auf zu den Konzepten von Ulla Otto auf, vor allem, was eine mögliche systemstabilisierende und instrumentale Funktion von Literatur betrifft.

${ }^{31}$ Hebel, 1980, S. 391-393.

${ }^{32}$ Hebel, 1980, S. 393; der Autor verweist auch auf Bronisław Malinowski, Szkice z teorii kultury. Książka i Wiedza, Warszawa 1958, S. 101-105. 
lem unterschiedlicher „Intentionen“ (Inkorporation, Unterordnung oder gar Bekämpfung) der dominierenden Kultur gegenüber alternativen Artikulationsformen der „anderen“ bzw. oppositionellen Kultur geht.

Einen Forschungsansatz, der dieses Problem in einer historischen Perspektive thematisiert und zugleich als methodologischer Vorschlag Perspektiven einer auf den rechtlichen Kontext orientierten Zensurforschung angesehen werden kann, repräsentiert Hubert Orłowski mit seinem auf eine „konfrontative Untersuchung von Zensursystemen" orientierten Aufsatz über die polnische Literatur unter preußischer und nationalsozialistischer Zensur ${ }^{33}$. Die Differenzen innerhalb rechtlicher Prozeduren im Wilhelminischen Deutschland und im Dritten Reich werden hier in einer engen Beziehung zu den jeweiligen allgemeinen politischen Zielen analysiert ${ }^{34}$. Der Vergleich zwischen den „schwarzen Listen“ aus der Wilhelminischen Zeit und denen aus der Zeit zwischen 1933 und 1945 weist auf deutliche Funktionsunterschiede der Zensuranordnungen hin, die den rechtlichen Kontext der einzelnen Maßnahmen bildeten. Selektivität und Systematik sind die beiden wichtigsten Unterschiede, auf die Orłowski in seinem Aufsatz aufmerksam macht:

„Wurde die polnische Literatur von der wilhelminischen Zensur wenn auch empfindlich, so doch nur gezielt-punktuell betroffen, so waren die Verbotslisten und -maßnahmen des Dritten Reiches ausdrücklich zu Instrumenten einer pauschalen Auslöschung der nationalpolnischen Literatur ausgebildet worden. Die wilhelminische Zensur sollte von der polnischen Bevölkerung öffentlich zur Kenntnis genommen werden, um nicht zuletzt dadurch den „öffentlichen Frieden“ abzusichern. Die Maßnahmen des Dritten Reiches dagegen wurden verheimlicht. Sie waren nicht auf Abschreckung berechnet, sollten nicht dem Erhalt eines wie auch immer problematischen ,äußeren Friedens“ dienen, sondern sie beabsichtigten die Vernichtung einer Nation als Kulturnation und damit die Vernichtung nationaler Identität. “35

Wir sprachen im Zusammenhang mit der „funktionalen“ Betrachtung des Problems der Zensurfolgen (Otto, Breuer) davon, daß die Zensur keinesfalls für die Eliminierung von Büchern und anderen Publikationen verantwortlich gemacht werden kann (wenn Bücher aus dem Kommunikationsumlauf „,verschwinden“, lautete die Hypothese, dann liegt der Grund dafür im Verlust ihrer sozialen Wirkung, und nicht in der Tätigkeit der Zensur). Politische Bedingungen, wie die, die den historischen Kontext der von Hubert Orlowski durchgeführten vergleichenden Analyse bildeten das Zensursystem im 3. Reich - markieren zweifelsohne die Grenzen der Gültigkeit dieser Hypothese. Dazu gehören auch Zensurentscheidungen, die demonstrativ außerhalb rechtlicher Festlegung getroffen werden konnten. Der deutsche Verwalter der Posener Bibliothek der „Freunde der Wissenschaften“, ein Beamter namens

\footnotetext{
33 Orłowski, 1988.

${ }^{34}$ ebda., S. $18 \mathrm{f}$.

35 ebda., S. 19.
} 
Dr. Scheif notierte 1940: „Ich kann diesen Haufen von Büchern vernichten, verbrennen; ich kann das tun, was mir Spaß macht.“36

Von hier aus führt ein nur sehr kurzer Weg zu einem „Endzustand“, den Bertolt Brecht in einem am Vorabend des 2. Weltkrieges geschrieben Gedicht seinen Lesern vor Augen geführt hat: Besuch bei den verbannten Dichtern - so lautet der Titel eines 1939 in Dänemark entstandenen Gedichts von Bertolt Brecht. Dieser Text vermittelt ein sehr komprimiertes Bild der Situation exilierter Schriftsteller, zugleich aber breitet es vor den Augen der Leser eine Art „Mind-Map“ der „Literaturzensur“ - vor allem im Hinblick auf ihre Folgen. Der in der „Hütte vertriebener Dichter“ ankommende Autor hört eine ,aus der dunkelsten Ecke“ kommende Stimme, die ihn fragt:

Du, wissen sie auch

Deine Verse auswendig? Und die sie wissen

Werden sie der Verfolgung entrinnen?.

Die Antwort, die Brecht in den Mund von Dante Alighieri legt, lautet:

Ihnen wurden nicht nur die Körper, auch die Werke vernichtet (...)

Keiner wagte hinüberzublicken.

Der Ankömmling

War erblaßt. ${ }^{37}$

\section{Zensur und kommunikative Textfunktion}

Literaturwissenschaftliche Zensurforschung, auf deren Positionen wir uns bis jetzt konzentriert haben, bezieht sich generell auf die Tätigkeit der Zensur, die das Funktionieren der Literatur in der Gesellschaft beeinflussen soll. Als Gründe und Motive gelten hier praktisch vor allem die von den Zensurinstanzen, von den einzelnen Zensurbeamten angenommenen, ja, ,erwarteten“"Wirkungen der Texte - es wäre daher durchaus gerechtfertigt, von einer Psychologie der Zensur (sprich: der Zensoren) zu sprechen.

Der Forschungsgegenstand - die potentielle Wirkung der Texte - weist auch auf Sprechakttheorien hin; gemeint ist hier vor allem der pragmatische Aspekt der Sprechakte. Der Sprechakt als Kreation sprachlicher Symbolik ist mit einem Zensurakt insofern vergleichbar als es sich auch bei dem Zensurakt um die Verwirklichung einer Sprechintention und die Konstruktion einer Welt sprachlicher Symbole, einer

\footnotetext{
36 ebda., S. 15 .

${ }^{37}$ Bertolt Brecht, Besuch bei den verbannten Dichtern. In: Brecht, B. 1997. Ausgewählte Werke in sechs Bänden, Suhrkamp Verlag, Frankfurt am Main, Bd. 3 (Svendborger Gedichte), S. 299 f.
} 
„Kommunikationssituation“ handelt, die in die vom zensierten Text potentiell ausgehende Kommunikation zumindest eingreift, sie einschränkt oder in extremen Fällen unmöglich macht. Dabei kann man in Anlehnung etwa an die Klassifikationsvorschläge von John R. Searle ${ }^{38}$ besonders auf Arten von „direktiven“ „kommissiven“ oder „deklarativen“ Sprechakten verweisen. Eine gewisse Grundstruktur bildet der direktive Sprechakt (oder hier auch der direktive Zensurakt), der auf die Formel „Schreibe nichts gegen den Staat“ bzw. „Du darfst nichts gegen den Staat schreiben“ zurückzuführen ist (wobei natürlich für den „Staat“ seine einzelnen Institutionen, Parteien, Wirtschafts- und Interessenverbände u. dgl. stehen können. Ein anderer Zensurakt oder ,Sprechakt der Zensur“ besteht in Situationen, in denen die Konsequenzen der Zensurperformanz als Gegenstand rechtlicher Vereinbarung erscheinen, wobei die Zensurinstanz über Möglichkeiten und Mittel verfügen muß, die (repressiven) Konsequenzen praktisch zu verwirklichen. Es handelt sich hier um einen Zensurakt, der als „kommissiver Akt“ bezeichnet werden kann. Er kann auf die Grundformel zurückgeführt werden die lautet „Wenn du etwas gegen den Staat schreibst, dann...". Ein deklarativer Zensurakt kann in der Mitteilung bestehen, die sich auf den „Endeffekt" des einzelnen Zensurkontrollmaßnahmen bezieht - es ist die Zustimmung zum Druck einer Publikation oder ihre Ablehnung (die entsprechenden formelhaften Äußerungen in diesem Fall wären etwa „ich stimme zu...“ bzw. ,ich lehne ab").

Hier soll jedoch angemerkt werden, (erstens) daß sich die von John R. Searle vorgeschlagene linguistische Theorie auf einzelne Aussagen und nicht auf komplexe geschweige denn literarische Texte bezieht. So behandelt Siegfried J. Schmidt in seiner Analyse Probleme einer Linguistik der sprachlichen Kommunikation (München 1973, S. 50 f.) den Text als eine „geordnete Anleitung“ an den Leser, der die im Text enthaltenen Informationen mit dem Ziel rezipiert, die vermittelten Informationen im Kontext sprachlicher und außersprachlicher (z.B. sozialer, politischer) Elemente zu verstehen.

Zweitens muß dazu auch noch angemerkt werden, daß eine derart „sprechakttheoretische" Konzeption der Zensurforschung eine eher idealtypische Konstruktion sein könnte und nicht alle Modalitäten und Faktoren umfassen kann, die sich auf Zensurentscheidungen auswirken bzw. ausgewirkt haben. Insbesondere muss sie mit der politologisch orientierten Zensurforschung (Dieter Breuer, Klaus Petersen, Hubert Orlowski, Michael Kienzle / Dirk Mende u.a.) nicht konkurrieren.

Was jedoch der „sprechakttheoretische“ Ansatz leisten kann, ist eine Rekonstruktion des Entscheidungs-Spielraums der Zensur und seiner psychosozialen und politischen Faktoren, vor deren Hintergrund die Zensurmaßnahmen als ,institutionelle Sprechakte“ getroffen wurden. Der Vorteil dieses Ansatzes liegt auch darin, daß hier nicht nur die Folgen der Zensurtätigkeit sondern auch der Text als geneti-

\footnotetext{
${ }^{38}$ Searle, 1969, S. 23.
} 
scher Proze $\beta^{39}$ in den Mittelpunkt rückt. Darüber hinaus ist die Diagnostizierung der von der Zensur getroffenen Maßnahmen unabhängig von der Form (von den sprachlichen Formen), in die diese Maßnahmen ,gekleidet“ wurden. So ist z.B. der Diskurs (die Form) eines „kollegialen Ratschlages“ wie er in der DDR oder im Dritten Reich - manchmal gar als „freundschaftlicher Rat" an einen Schriftsteller gerichtet werden konnte, von dem betreffenden (oder eigentlich betroffenen) Schriftstellerkollegen sicherlich unmißverständlich als keinen Widerstand duldende Empfehlung, Anweisung, Befehl oder Warnung den entsprechenden semantischen Feldern zugeordnet worden. Joachim Walther weist in seiner Dokumentation Sicherungsbereich Literatur. Schriftsteller und Staatssicherheit in der Deutschen Demokratischen Republik (Berlin 1996) auf diese „Vielfalt“ möglicher funktionaler Zuordnungen von Äußerungen und Maßnahmen hin. Im Bereich der Kontakte der DDR-Schriftstellern zu den Institutionen der politischen Kontrolle (oder der „Kulturpolitik im allgemeinen) spricht Walther vom „Methodenbündel“, d.h. von einer Mischung aus Persuasion, Belehrung, Überredung, positiver oder negativer Sanktionierung, Manipulation, und anderen Strategien, die die DDR-Intellektuellen beeinflussen sollten. ${ }^{40}$

In den Dokumenten des Staatssicherheitsdienstes der DDR gibt es unter anderem eine Empfehlung für „politisch-operative Arbeit“ der IMs. Dort ist unter anderem zu lesen:

„Um nicht vordergründig auf die Vorgangsperson politisch-ideologisch einzuwirken, müssen verschiedene inoffizielle Quellen in abgestimmter Form aus unterschiedlichen gesellschaftlichen Bereichen eingesetzt werden. Um dies deutlich zu machen: Es soll im Sinne der Neutralisierung / Rückgewinnung auf eine international bekannte Persönlichkeit Einfluß genommen werden, die zum Beispiel - Mitglied der Akademie der Künste der DDR, - Mitglied eines Künstlerverbandes der DDR, - Mitglied einer internationalen Vereinigung ist. Hier ist es notwendig, daß IM aus folgenden Bereichen auf diese Person einwirken: - ein geeigneter IM aus dem zuständigen staatlichen Fachbereich, der die Vorgangsperson in seiner beruflichen Arbeit betreut und Einfluß darauf nimmt, daß in der künstlerischen Arbeit der Vorgangsperson keine Probleme gestaltet werden, die politisch-ideologisch einer Veröffentlichung in der DDR entgegenstehen, - ein weiterer IM muß sich darum bemühen, diese Vorgangsperson in die Arbeit der Akademie der Künste der DDR einzubeziehen usw. ${ }^{41}$

Ein solches Vorgehen wurde in den 70er Jahren unter anderem gegen Franz Fühmann eingesetzt; dies jedoch ohne der gewünschten Effekt.

${ }^{39}$ Hier ist die Unterscheidung zwischen textbezogenen und extratextualen Folgen der Zensur und (auch) der Selbstzensur wichtig. Die Selbstzensur als spezifisches „Zensurprodukt“, das dem Staat ermöglicht, sich im Schein der Meinungsfreiheit zu „sonnen“, ist ein weiteres Element, das in die methodologische Reflexion der Zensurforschung einbezogen wird. Siehe auch Wolfgang Emmerich, Heinrich Mann „Der Untertan”, München 1980; auch Kanzog, K. 1988, S. 324 f.

${ }^{40}$ Walther, 1996, S. 352.

${ }^{41}$ Walther, op. cit. S. $354 \mathrm{f}$. 
Hier sehen wir auch, dass noch ein weiterer, möglicherweise interdisziplinär orientierter Aspekt der Zensurforschung interessante Erkenntnisse liefern könnte: es ist der psychologische Aspekt der Zensur; insbesondere die Frage nach einer „Psychologie des Zensors”, die möglichst alle Aspekte des erwähnten „Methodenbündels“ erfassen könnte.

\section{Sekundärliteratur}

Assmann, A. und J. Assmann. 1987. Kanon und Zensur. Beiträge zur Archäologie der literarischen Kommunikation II. München: Wilhelm Fink Verlag.

Bark, S., Lokatis, S. u.a. 1997. Jedes Buch ein Abenteuer. Zensur-System und literarische Öffentlichkeit(en) in der DDR bis Ende der 60er Jahre. Berlin: Zentrum für Zeithistorische Forschung Potsdam.

Bartels, A. 1942. Geschichte der deutschen Literatur. 18. Aufl. Braunschweig, Berlin, Hamburg: Westermann.

Biermann, A. 1987. „Zur sozialen Konstruktion der 'Gefährlichkeit' von Literatur. Beispiele aus der französischen Aufklärung und dem Premier Empire“. In: Assmann. 212-226.

Brecht, B. 1997. Ausgewählte Werke in sechs Bänden. Bd. 3 (Svendborger Gedichte). Frankfurt a. M.: Suhrkamp Verlag. 299f.

Breuer, D. 1982 Geschichte der literarischen Zensur in Deutschland. Heidelberg: Quelle und Meyer.

Brockmeier, P. 1996. (Hrsg.). Kaiser, Gerhard R.: Zensur und Selbstzensur in der Literatur. Würzburg: Verlag Königshausen und Neumann.

Denk, F. 1995. Die Zensur der Nachgeborenen. Zur regimekritischen Literatur im Dritten Reich. Weilheim i. OB: Denk-Verlag.

Eisenhardt, U. 1988. „Wandlungen von Zweck und Methoden der Zensur im 18. und 19. Jahrhundert“. In: Göpfert und Weyrauch. 1-35.

Emrich, W. 1968. „Kann die Jugend durch Literatur 'gefährdet' werden?“. In: Emrich, W. Polemik. Streitschriften, Pressefehden und kritische Essays um Prinzipien, Methoden und Maßstäbe der Literaturkritik. Frankfurt a.M.: Athenäum Verlag. 215-220.

Fechter P. 1941. Geschichte der deutschen Literatur. Von den Anfängen bis zur Gegenwart. Berlin.

Göpfert, H.G. und E. Weyrauch. 1988. "Unmoralisch an sich ...": Zensur im 18. und 19. Jahrhundert. [Vorträge des 7. Jahrestreffens des Wolfenbütteler Arbeitskreises für Geschichte des Buchwesens vom 14. bis 17. Mai 1985 in der Herzog August Bibliothek].

Hebel, F. 1980. „Die Rolle der Literatur in der Kulturvermittlung oder: Gibt es eine 'repräsentative Relevanz` von Texten: Erörtert am Beispiel von Gerhart Hauptmanns Komödie "Der Biberpelz"“. In: Fremdsprache Deutsch 2. Grundlagen und Verfahren der Germanistik als Fremdsprachenphilologie Bd. 1, hrsg. von Alois Wierlacher. München: Wilhelm Fink Verlag. 387-409.

Hobohm, H.-C. 1992. Roman und Zensur zu Beginn der Moderne. Vermessung eines sozio-poetischen Raumes. Paris 1730-1744. Frankfurt a. M.: Campus-Verlag.

Houben, H. 1965. Verbotene Literatur von der klassischen Zeit bis zur Gegenwart. Ein kritischhistorisches Lexikon über verbotene Bücher, Zeitschriften und Theaterstücke, Schriftsteller und Verleger. Hildesheim, (2 Bde.). Nachdruck der Ausgabe Berlin 1924 (Bd. 1), 1928 (Bd. 2).

ders. 1978. Der ewige Zensor. Längs- und Querschnitte durch die Geschichte der Buch- und Theaterzensur. Nachdruck der Ausgabe von 1926. Kronberg/Ts.: Athenäum-Verlag.

ders. 1918. Hier Zensur - wer dort? Antworten von gestern auf Fragen von heute. Leipzig: F.A. Brockhaus. 
Kanzog, K. 1984. „Zensur, literarische“. In: Kazog, K. et al. (Hrsg.). Reallexikon der deutschen Literaturgeschichte, Bd. 4, hrsg. von Klaus Kanzog und Achim Masser. Berlin: De Gruyter. 998-1049.

Kanzog, K. 1988. „Textkritische Probleme der literarischen Zensur. Zukünftige Aufgaben einer literaturwissenschaftlichen Zensurforschung“. In: Göpfert und Weyrauch. 309-331.

Karolak, C. 2000 (Hrsg.). Cenzura w Niemczech w XX wieku. Studia, analizy, dokumenty. Poznań: Wydawnictwo Poznańskie.

Ketelsen, U. 1992. Literatur und Drittes Reich. Schernfeld: SH-Verlag (Süddeutsche Hochschulverlagsgesellschaft).

Kienzle, M. und D. Mende. 1980. Zensur in der BRD. Fakten und Analysen. München: Carl Hanser Verlag.

Koch, F. 1935. Deutsche Kultur des Idealismus. Potsdam: Akademische Verlagsgesellschaft Athenaion M. B. H.

ders. 1935. Klassik und Romantik der Deutschen. Frankfurt a.M.

Linden, W. 1940. Geschichte der deutschen Literatur von den Anfängen bis zur Gegenwart. Leipzig (2. erw. Aufl.): Phillip Reclam.

Lübbe, F. und H.F. Lohrmann. 1943. Deutsche Dichtung in Vergangenheit und Gegenwart. Ein Führer durch die deutsche Literatur. 4 Aufl. Hannover.

Malinowski, B. 1958. Szkice z teorii kultury. Warszawa: Książka i Wiedza.

Orłowski, H. 1983. „Zu einigen `formalen` Aspekten der Literaturgeschichtsschreibung im Zeichen des deutschen Faschismus“. In: Hartung, G. Traditionen und Traditionssuche des deutschen Faschismus, Bd. 1. Halle.

Orłowski, H. 1988. Polnisches Schrifttum unter Zensur. Wilhelminische und nationalsozialistische Zensurpolitik im Vergleich. Frankfurt a.M.: Verlag Moritz Diesterweg.

Otto, U. 1968. Die literarische Zensur als Problem der Soziologie der Politik. Stuttgart: Enke-Verlag.

Petersen, K. 1988. Literatur und Justiz in der Weimarer Republik. Stuttgart: J.B. Metzlersche Verlagsbuchhandlung.

ders. 1995. Zensur in der Weimarer Republik. Stuttgart, Weimar: Verlag J.B. Metzler.

Schütz, H.J. 1990. Verbotene Bücher. Eine Geschichte der Zensur von Homer bis Henry Miller. München: C.H. Beck'sche Verlagsbuchhandlung.

Searle, J.R. 1969. Speech acts. An essay in the philosophy of language. Cambridge: Cambridge Univerity Press.

Tullock, G. 1968. „A note on cenzorship“. America Political Science Review 62. 1265-1267.

Walther, J. 1996. Sicherungsbereich Literatur. Schriftsteller und Staatssicherheit in derDeutschen Demokratischen Republik. Berlin: . Ch. Links Verlag.

Wieland, Chr.M. 1967. „Das Geheimnis des Kosmopolitenordens“. In: Wieland. Werke. Hrsg. Von Fritz Martini und Hans Werner Seiffert, Bd. 3, bearbeitet von Fritz Martini und Reinhard Döhl. München: Carl Hanser Verlag. 573 f. 